Onomástica Desde América Latina, n.4, v.2, julho - dezembro, 2021, p.95-109.ISSN 26752719

https://doi.org/10.48075/odal.v0i0.26567

\title{
Etude d'un échantillon patronymique algérien : Exemple d'un calque identitaire à Mostaganem
}

\section{Study of an Algerian surname sample: Example of erasing of identity in Mostaganem}

\author{
Soufiane Bengoua \\ Université Abd Elhamid Ibn Badis-Mostaganem, Algérie \\ https://orcid.org/0000-0002-3763-8353 \\ soufiane.bengoua@univ-mosta.dz
}

\section{Résumé}

Notre intervention cadre volontiers avec la thématique de la revue. Nous optons pour une analyse lexico-sémantique d'un échantillon de patronymes français et mostaganémois de 1900 à $1903^{1}$, période qui a suivi l'instauration du code de l'indigénat entre 1873 et 1883 . Cette analyse va mettre en relief la politique d'effacement identitaire entreprise par le colonisateur. Notre démarche dans le cadre de cette contribution consiste à illustrer le mode d'attribution des patronymes algériens selon une structure déjà en place en France et qui demeure aux antipodes de la tradition onomastique traditionnelle maghrébine. En effet, à travers une lecture comparative entre une série de patronymes choisis sur un site ${ }^{2}$ spécialisé dans les noms de famille français et une autre série récoltée par nous dans une recherche antérieure (BENGOUA \& MALEK, 2001), nous allons mettre en exergue cette volonté délibérée du colonisateur de balayer une identité arabo-musulmane déjà en place via une déstructuration onomastique maghrébine sur un modèle onomastique français.

Mots-clés : patronymes français, patronymes mostaganémois, effacement identitaire, déstructuration onomastique, restructuration onomastique.

\begin{abstract}
Our intervention fits readily with the theme of the review. We opt for a lexico-semantic analysis of a sample of French and Mostaganémois surnames from 1900 to 1903, the period which followed the establishment of the code of the native population between 1873 and 1883. This analysis will highlight the policy of identity erasure undertaken by the colonizer.

Our approach within the framework of this contribution consists in illustrating the mode of attribution of Algerian surnames according to a structure already in place in France and which remains at the antipodes of the traditional Maghreb onomastic tradition. Indeed, through a comparative reading between a series of surnames chosen on a site specializing in French surnames and another series collected by us in previous research, we will highlight this deliberate desire of the colonizer to sweep away an Arab-Muslim identity already in place via a Maghrebian onomastic destructuring on a French onomastic model.
\end{abstract}

\footnotetext{
${ }^{1}$ C'est l'échantillon d'un corpus recueilli dans le cadre de notre mémoire de licence

${ }^{2} \mathrm{http}: / / \mathrm{www}$.journaldesfemmes.com/nom-de-famille/noms/1/13/france.shtml
} 
Onomástica Desde América Latina, n.4, v.2, julho - dezembro, 2021, p.95-109.ISSN 26752719

https://doi.org/10.48075/odal.v0i0.26567

Keywords: French surnames, Mostaganémois surnames, identity erasure, onomastic destructuring, onomastic restructuring.

\section{Introduction}

Les patronymes font partie de notre patrimoine linguistique au même titre que les mots du vocabulaire. La configuration patronymique actuelle n'est qu'une expression d'une volonté délibérée de détruire le pilier vital de l'identité algérienne opérant ainsi une rupture généalogique inscrite dans la lignée arabo-musulmane. Spolier le bien immatériel de l'algérien demeure la priorité absolue du colonisateur français. En effet, la stratégie opérée ne s'est pas limitée à l'assujettissement physique à travers les violations de territoires, mais elle a adopté un asservissement identitaire et onomastique pour que l'autochtone soit assimilé à la culture française. L'attribution d'un patronyme qui se substitue à ceux régis par les traditions onomastiques maghrébines ne fait qu'ébranler cette collectivité algérienne et élimine des ressources identitaires authentiques prises dans la pluralité de notre patrimoine historique, culturel et linguistique.

La stratégie coloniale a voulu franciser le peuple algérien corps et âme en le dénudant de son héritage religieux, culturel, linguistique et anthroponymique. «La principale erreur commise en Afrique du Nord consiste à vouloir y appliquer les principes politiques et administratifs qui régissent la France du XXème siècle »(LE COUR GRANDMAISON, 2010 :9), soutient en 1925 le général Paul Azan militaire et historien colonial du début du XXème siècle. Le colonialisme pratiqué en Algérie s'apparentait à une sorte d'esclavage de la population autochtone, dépouillée de toute son identité.

Les Mostaganémois dont les noms sont l'objet de cet article avaient eux aussi subi les vicissitudes de la loi 1882, et ont dû délaisser malgré eux un héritage onomastique séculaire. Nous savons que la ville de Mostaganem était le creuset de différents passages (Phéniciens, Romains, Espagnols, Arabes, Turcs et Français) qui ont versé à la ville leur culture, leur 
Onomástica Desde América Latina, n.4, v.2, julho - dezembro, 2021, p.95-109.ISSN 26752719

https://doi.org/10.48075/odal.v0i0.26567

patrimoine et leur langue. En fait, le colonisateur de par cette opération qui ciblait l'intégration

du peuple algérien aux Français a su transplanter un greffon difficilement supporté dans la structure identitaire de l'Algérien, à savoir son patronyme. En effet, nous avons remarqué que presque $50 \%$ des patronymes mostaganémois intègrent une base anthroponymique relative à la tradition onomastique maghrébine. L'étude que nous avons faite, nous a révélé que l'élément sui generis du patronyme mostaganémois se caractérise comme suit :

1. Presque plus de $80 \%$ des noms de famille s'inscrivent dans le lexique algérien de la région de Mostaganem.

2. Les bases anthroponymiques de la tradition onomastique maghrébine y sont présentes (BEN- $42 \%$, BOU- $7.4 \%$ et BEL- $9 \%$ )

3. Trois catégories sémantiques sont dominantes :

a- Les noms de prophètes $(4.5 \%)$

b- Les noms du prophète de l'islam (4.3\%)

c- Les noms des saints locaux $(3.2 \%)$

4. Les variantes graphiques pour désigner [غं] et [خ்]

Exemple : BEGHDADI et BERDADI.

\section{KHALIFA et KRELIFA}

La loi de 1882, qui n'est que la suite de celle de 1873 sur la propriété individuelle, imposait l'adjonction d'un patronyme au prénom et surnom par lesquels était antérieurement connu chaque indigène déclaré propriétaire. La quintessence de cette loi était l'imposition d'un système de nomination calqué sur le modèle français.

Nous ne pouvons manquer de rappeler que l'attribution d'un patronyme qui s'apparenterait beaucoup plus au système de nomination français ne s'est pas fait sans le concours d'autochtones et d'interprètes étrangers comme les Levantins et les Juifs. Nous avons vu dans notre étude effectuée sur les patronymes mostaganémois que $81.8 \%$ des noms de 
Onomástica Desde América Latina, n.4, v.2, julho - dezembro, 2021, p.95-109.ISSN 26752719

https://doi.org/10.48075/odal.v0i0.26567

famille sont issus de l'arabe dialectal(BENGOUA. S \& MALEK, 2005). Cette dominante dialectale a été sciemment intégrée par un système qui maîtrise la langue autochtone, à savoir la variante locale de l'arabe. En outre, nous savons que les Français étaient soucieux de conquérir l'Algérie sans connaissance aucune des traditions du pays et surtout sans la maitrise des dialectes algériens. C'est pour cette raison qu'ils ont fait appel à des interprètes.

Au cours de cette contribution, nous essayerons de mettre en relief cette stratégie d'assujettissement via la comparaison de deux corpus de patronymes français et mostaganémois. Nous nous demandons si les patronymes mostaganémois ressemblent aux noms français et, si oui, quels en sont les points communs. Y aurait-il une traduction systématique des noms de famille mostaganémois?

A travers une approche lexico-sémantique, nous montrerons que les noms mostaganémois ont été calqués sur un modèle français qui n'a aucun lien avec le système onomastique maghrébin.

\section{Présentation et analyse du corpus}

Dans le cadre de cette contribution, nous allons passer en revue 247 noms français et analyser 88 noms algériens (de Mostaganem) pour mettre en avant la politique onomastique imposée aux Algériens à travers la loi de 1882 instaurée par la France, afin de montrer du doigt le calque des patronymes algériens, et mostaganémois en particulier, effectué sur le modèle onomastique français. Pour cela, nous avons répertorié des noms français récoltés sur le site Journal des femmes ${ }^{3}$ qui se base sur son service de retrouvailles Copains d'avant qui capitalise 11 millions de Français inscrits pour retrouver des amis perdus de vue, en indiquant notamment leur nom de famille et leur lieu de résidence. Nous avons aussi inventorié des 
Onomástica Desde América Latina, n.4, v.2, julho - dezembro, 2021, p.95-109.ISSN 26752719

https://doi.org/10.48075/odal.v0i0.26567.

patronymes mostaganémois relevés directement sur les registres d'état civil lors d'une enquête

de terrain ${ }^{4}$.

Dans un premier moment, nous allons décrire les différentes catégories de noms français inventoriés, puis nous procédons à la comparaison avec les patronymes mostaganémois que nous avons recueillis.

\subsection{Inventaire des noms français morphologiquement simples (102 noms)}

\begin{tabular}{ll}
\hline NOMS de & PATRONYMES \\
\hline Prophètes & ADAM, NOE, JACOB, DAVID, MOISE, JESUS \\
\hline Saints locaux & ETIENNE, BERNARD \\
\hline Animaux & POISSON, MOUCHE, CHEVAL, MULLET, CHAT, CHIEN. \\
Parenté & COUSIN, NEVEU, GENDRE, ONCLE, PARENT \\
Fêtes religieuses & TOUSSAINT, NOEL, PAQUES. \\
Métiers & CHARBONNIER, POTIER, LARCHER, PORTIER, MENUISIER, FORGERON, \\
\end{tabular}

\section{Tableau1}

\begin{tabular}{ll}
\hline NOMS de & PATRONYMES \\
\hline D'endroits & GRENIER, CUISINE, SALON, CHAMBRE, GRANGE. \\
\hline D'objets & BOULET, MARTEAU, RIVET, CLOCHE, SOULIER, COLLIER, BOUTON.
\end{tabular}

\begin{tabular}{l|l}
\hline Relatifs à la nature & ROCHER, RIVIERE, MONTAGNE, VALLON. \\
\hline Fonctions et titres honorifiques & MAIRE, COMPTE, PRINCE, DUC. \\
\hline Fruitiers & POMMIER, CERISIER, BANANIER, POIRIER, FIGUIER, PECHER. \\
\hline D’appartenance géographique & GALLOIS, BRETON. \\
\hline
\end{tabular}

\footnotetext{
${ }^{4}$ Recueil de données effectué lors de notre enquête en 2000 pour notre mémoire de fin de licence sur les patronymes mostaganémois de 1900 à 1903.
} 
Onomástica Desde América Latina, n.4, v.2, julho - dezembro, 2021, p.95-109.ISSN 26752719

https://doi.org/10.48075/odal.v0i0.26567

\begin{tabular}{l|l}
\hline Relatifs au corps humain & $\begin{array}{l}\text { SANG, NEZ, FRONT, PIED, BRAS, MENTON, GENOU, VISAGE, TALON, CUL, } \\
\text { MOUSTACHE, BARBE }\end{array}$ \\
\hline Excréments & MERDE, BOUSE.
\end{tabular}

\begin{tabular}{l|l} 
Végétaux & FLEUR, ARBRE, ROSIER, LILAS, LYS, FOUGERE, TULIPE, HOUX, PLATANE. \\
\hline Ustensiles de cuisine & COUTEAU, MARMITE, LOUCHE, TABLE.
\end{tabular}

\begin{tabular}{l|l} 
Instruments de musique & PIANO, VIOLON, TAMBOUR, FLUTE. \\
\hline Relatifs à une région & PARIS, CHAMPAGNE, BORDEAU, MARSEILLE, MIDI, LILLE, ALBI
\end{tabular}

Relatifs à une région PARIS, CHAMPAGNE, BORDEAU, MARSEILLE, MIDI, LILLE, ALBI.

\begin{tabular}{l|l}
\hline Oiseaux et volatiles & CANARD, CORBEAU, PIGEON, COLOMBE, CIGOGNE. \\
\hline
\end{tabular}

Tableau2

\subsubsection{Lecture tabulaire}

A travers cet inventaire, de nouvelles catégories émergent. En effet, nous remarquons que sur les 100 noms simples morphologiquement, $100 \%$ sont des substantifs masculins désignant 19 catégories différentes allant des noms de prophètes ou d'objets jusqu'aux noms d'arbres ou de métiers. Notons au passage que la tradition onomastique française compte quatre catégories lexicales (MIYAMATSU,2002 :106) de noms de famille :

- Des noms qui correspondent à des noms de baptême, noms du chef de famille d'origine.

- Des noms qui désignent un lieu, site d'origine ou de la localisation de la famille.

- Des noms qui traduisent un métier du premier porteur du patronyme.

- Des noms qui traduisent un sobriquet reflétant un lien de parenté, une particularité physique ou morale parfois désignée par un nom d'animal.

Les mêmes patronymes relatifs aux noms d'animaux qui ont longtemps été portés fièrement par les Français, sont utilisés aujourd'hui à des fins politiques. Nous pouvons lire dans la tribune de LeParisien la proposition d'une loi, un peu banale «Jean-Frédéric Poisson, 
Onomástica Desde América Latina, n.4, v.2, julho - dezembro, 2021, p.95-109.ISSN 26752719

https://doi.org/10.48075/odal.v0i0.26567

le député $(P C D)$ de Rambouillet, Jean-Marie Tétart, son voisin des Yvelines et Franck Marlin

veulent détendre l'atmosphère des bancs de l'Assemblée nationale en apportant un moment de fantaisie. Ils entendent se servir de leurs noms pour faire une boutade. Ils prévoient de déposer une proposition de loi le ler avril pour protéger les députés qui portent un nom d'animal aquatique. $»^{5}$

Malgré la multitude de catégories lexicales des patronymes français leur morphologie est homogène. En effet, tous les patronymes sont morphologiquement simples et sont au singulier. Nous les retrouvons collés à une ou à deux particules comme dans RUE, LARUE, DELARUE.

2.2 Inventaire des noms français composés de déterminants ${ }^{6}$ ou « particules » (DE-, DU, LE-, LA-, DES-) (98 noms)

Avant de présenter les différents patronymes composés, nous précisons que nous avons écarté les noms dits des nobles à savoir les noms dont la particule nobiliaire DE est séparée du nom par un blanc. Nous notons aussi que l'intégralité des noms composés des cinq particules, à savoir DE, DU, LE, LA et DES, existe aussi sans particule. Ainsi, nous citons à titre d'exemple DELANNOY, LANNOY, DELAPORTE, LAPORTE, PORTE.

\footnotetext{
Particules Patronymes

${ }^{5} \mathrm{http}: / /$ www.leparisien.fr/houdan-78550/les-deputes-deposeront-une-proposition-de-loi-pour-rire-08-01-20132462491.php(site consulté le 19/09/2018 à 14h00)

${ }^{6}$ En langue familière, on parle de «noms à particule» pour signaler ce rattachement des porteurs à la noblesse. Les particules les plus courantes sont «de », «d'», « de la », « du » et « des ».
} 
Onomástica Desde América Latina, n.4, v.2, julho - dezembro, 2021, p.95-109.ISSN 26752719

https://doi.org/10.48075/odal.v0i0.26567

\begin{tabular}{ll}
\hline \hline DE+ & DE + LANNOY, DE+LAHAYE, DE+LAPORTE, DE+SOUSA, \\
& DE+OLIVEIRA, DE+LARUE. \\
& \\
DU+ & DU+BOIS, DURAND, DURANG, DURANT, DUPONT, DUPOND, \\
& DUMONT, DUVAL, DUFOUR, DUPUIS, DUHAMEL, DUBREUIL, \\
& DUCHEMIN, DUMOULIN, DUJARDIN, DUCLOS, DUBUS, \\
& DURIEUX, DUPRAT, DUCHENE, DUCHESNE, DUCROCQ.
\end{tabular}

$\begin{array}{ll}\text { LE+ } & \text { LE+GUEN, LEROY, LEFEVRE, LEFEUVRE, LEGRAND, } \\ & \text { LEMAIRE, LEROUX, LECLERC, LEMOINE, LECLERE, } \\ & \text { LECLERCQ, LEGALL, LECOMTE, LEGOFF, LEBRUN, } \\ & \text { LEVEQUE, LESAGE, LEBLANC, LEMAITRE, LEGROS, } \\ & \text { LEJEUNE, LEDUC, LEBRETON, LEGENDRE, LEFORT, LENOIR, } \\ & \text { LECONTE, LELIEVRE, LEBLOND, LECOQ, LEMONNIER, } \\ & \text { LECORRE, LELONG, LEBON, LECOCQ, LELEU, LEBRIS, } \\ & \text { LEFRANCOIS, LEBRAS, LEDOUX, LERAY, LEBERRE, LEBER, } \\ & \text { LEBEAU, LECUYER, LEPAGE, LEVASSEUR, LEBIHAN, } \\ & \text { LEBORGNE, LEGAL, LEFRANC. }\end{array}$

$\mathbf{L A +}$

LA+CROIX, LAPORTE，LACOMBE，LAROCHE，LAGARDE, LABORDE, LACOSTE, LAFON, LACOUR, LAVAL, LALAUNE, LAVIGNE, LATOUR, LASSERRE, LAPIERRE.

DES+ DES+ CHAMPS, DESCAMPS, DESBOIS, DESHAYES

Tableau3

\subsubsection{Lecture tabulaire}

Nous remarquons que sur les 98 noms morphologiquement composés, ceux composés de la particule LE atteignent une fréquence de $52.04 \%$, les noms en DU $22.44 \%$, ceux en LA $15.30 \%$, et les noms en DE 6.12\%. Enfin, les noms en DES représentent $4.08 \%$.

Signalons au passage que pas tous les porteurs d'un nom de famille de ce genre n'appartiennent à la noblesse. D'ailleurs, on dit souvent, Gens à particule, c'est-à-dire des personnes dont le nom de famille usuel est précédé de la particule DE, laquelle n'est pas toujours une marque originelle de noblesse. Nous pouvons citer à titre d'exemple un passage tiré du roman Les célibataires d'Henri de Montherlant « Ce qu'il visait toujours, c'était la particule, selon l'usage 
Onomástica Desde América Latina, n.4, v.2, julho - dezembro, 2021, p.95-109.ISSN 26752719 https://doi.org/10.48075/odal.v0i0.26567

du peuple, qui ignore que, autant il y a de gens à particule, et qui ne sont pas nobles, autant (ou presque) il y a de gens qui ne l'ont pas, et qui le sont »(DEMONTHERLANT, 1934 :.184).

L'ensemble des noms du tableau se compose d'une particule + un substantif ou un adjectif. Nous pouvons détailler comme suit :

LE+ substantif (titre honorifique, appartenance géographique, nom d'animal)

LE+ adjectif (qualité physique, qualité morale)

LA+ substantif (élément de la nature, objet)

DES+ substantif au pluriel (élément de la nature)

DE+ substantif (élément de la nature, objet, nom d'une personne)

Sur le plan sémantique, les particules jouent un rôle déterminant et variable à la fois. En d'autres termes, la particule «LE » s'agglutine au substantif ou à un adjectif pour définir le patronyme avec une seule signification spécifique, comme un métier ou une particularité physique ou morale, tandis que les particules « DU, DES, LA, DE » ont une signification variable. En effet, si nous prenons par exemple DUPONT ou DUMOULIN, nous constatons que DU est plutôt synonyme de «près de » ou «celui à ». Cependant, il faudrait rappeler que des noms de ce genre ont été attribués à des enfants trouvés sous le pont ou près du moulin.

Nous distinguons, de par notre inventaire, 6 catégories de patronymes morphologiquement composés :

$\mathrm{DE}+$ nom simple, $\mathrm{DU}+$ nom simple, $\mathrm{LE}+$ nom simple, $\mathrm{LA}+$ nom simple, $\mathrm{DE}+\mathrm{LA}+$ nom simple, DES+ nom simple. 
Onomástica Desde América Latina, n.4, v.2, julho - dezembro, 2021, p.95-109.ISSN 26752719

https://doi.org/10.48075/odal.v0i0.26567

Le même procédé a été utilisé pour les bases anthroponymiques ou les particules de filiation du patronyme mostaganémois, BEN et BOU. Initialement, elles sont considérées comme l'élément sui generis de la structure onomastique maghrébine avec des significations stables et monosémiques renvoyant respectivement à $\mathrm{BEN}$ « fils de » et BOU «père de », mais il en est une réalité toute autre.

- BEN acquiert une autre signification

« Celui à » BENKHOUDMI, celui au couteau.

«Fils de » BENYAHIA, Yahia dans le Coran est le synonyme de « Jean » dans les évangiles.

«Celui qui » BENSEKKOUMA = "Celui à l'asperge"

- BOU acquiert une autre signification

« Celui au » BOUKHOUDMI, celui au couteau.

« Père de » BOUZID, en Orient «Zayd » est un prénom très utilisé, car il renvoie au prénom du compagnon du prophète « Zayd Ibn Harithah ».

«Celui qui » BOUKRA, celui à une seule jambe ou l'unijambiste.

Il n'est pas rare de trouver deux bases anthroponymiques juxtaposées. Dans le patronyme BENBOUZIANE par exemple, nous retrouvons ainsi le grand-père «Bouziane », le père «Ziane» et le fils «Benbouziane ». Les deux bases anthroponymiques catalysent la chaine originelle de filiation.

\subsection{Inventaire de noms ayant subi une agglutination}

DUPOND, DUPONT, LALLEMAND, LALLEMENT, DURANT, DURANG, DURAND, LANGLAIS. 
Onomástica Desde América Latina, n.4, v.2, julho - dezembro, 2021, p.95-109.ISSN 26752719

https://doi.org/10.48075/odal.v0i0.26567

Nous remarquons que non seulement dans les 8 patronymes ci-dessus, il y a présence d'une particule qui s'est agglutinée au patronyme, mais en plus, il y a des variantes graphiques de deux noms:

*DUPOND / DUPONT (même prononciation /oralisation, mais variation graphique)

*LALLEMAND / LALLEMENT (variante de transcription phonétique)

*DURANT/ DURANG/ DURAND (variantes de transcription phonétique)

Nous constatons que cette variation graphique dans les patronymes français fait partie intégrante de la structure onomastique. Cependant, si nous la transposons aux patronymes algériens et mostaganémois en particulier, nous remarquons une déconstruction flagrante. Nous pouvons citer à titre d'exemple les patronymes :

*BENYEKHOU/ BENYAKKOU (confusion entre $[\chi]$ et $[\mathrm{k}]$ )

*BENCHIDA/BENCHEHIDA (absence du [^])

* LAZREG/LAZREUG (la voyelle mi-ouverte écartée $[\varepsilon]$ devient mi-ouverte arrondie [œ]en syllabe fermée)

/BENAIMEUR/ BENAMEUR/BENAOUMEUR (fausses diphtongues entre /ay/ /aw/)

Nous retrouvons ici l'illustration de la volonté d'annihiler les Algériens qui ont été « indignement lésés par les anciens services coloniaux »( LACHERAF, 1998 : 168).

2.4 Inventaire des noms simples morphologiquement (81 noms mostaganémois+ 41 noms français) 
Onomástica Desde América Latina, n.4, v.2, julho - dezembro, 2021, p.95-109.ISSN 26752719

https://doi.org/10.48075/odal.v0i0.26567

\begin{tabular}{|c|c|c|}
\hline Noms mostaganémois & Noms français & caractéristiques communes \\
\hline FARES/ BENFARES & CAVALIER & synonyme \\
\hline HADJ/ BELHADJ & PELERIN & synonyme \\
\hline BERBER & CORSE, BRETON & origine ethnique \\
\hline OUALI/ BENOUALI & SAINT & synonyme \\
\hline CHERIF/ BENCHERIF & SAINT & synonyme \\
\hline OUZAA/ BENOUAZA & CANARD & oiseau palmipède \\
\hline OUAHBA & DON & synonyme \\
\hline GHANI/ BELGHALI & RICHE & synonyme \\
\hline SALAH/ BENSALAH & VERTUEUX, BON & synonyme \\
\hline TOUIL/ BETOUIL & GRAND & synonyme \\
\hline RABAH/ BENRABAH & GAGNANT & synonyme \\
\hline GHANEMI & BUTIN & synonyme \\
\hline MAGHNIA/ BELMAGHNI & PARIS/ ALBI & zone géographique \\
\hline MEBAREK & BENI & synonyme \\
\hline ESSAHLI & COTIER & synonyme \\
\hline LAZREUG/ BELAZREUG & LEBLEU & synonyme \\
\hline MEKKI/ BELMEKKI & LOURDAIS & relatif à un lieu saint \\
\hline REGUIG & MAIGRE & synonyme \\
\hline BEHRI & MARIN & synonyme \\
\hline AFIF & CANDIDE & synonyme \\
\hline BARKA & ARRET & synonyme \\
\hline
\end{tabular}

Tableau4 
Onomástica Desde América Latina, n.4, v.2, julho - dezembro, 2021, p.95-109.ISSN 26752719

https://doi.org/10.48075/odal.v0i0.26567

\begin{tabular}{|c|c|c|}
\hline ZITOUNI & OLIVIER & synonyme \\
\hline SABEUR/ BENSABEUR & PATIENT & synonyme \\
\hline MOUSSA/ BENMOUSSA & MOISE & synonyme \\
\hline DJEDDOU/ BENDJEDDOU & PAPI & synonyme \\
\hline KOUSKESA & MARMITE & noms d'ustensiles \\
\hline DAOUD/ BOUDAOUD & DAVID & synonyme \\
\hline SERIR/ BENSERIR & LIT & synonyme \\
\hline ARBI/ BELARBI & FRANCCAIS & appartenance ethnique \\
\hline $\mathrm{CHAIB} /$ & $\hat{A} G E ́$ & synonyme \\
\hline BENCHAIB/BOUCHAIB & & \\
\hline LATROCH/ BELATROCHE & SOURD & synonyme \\
\hline YACOUBI & JACOB, JACOBI & synonyme \\
\hline DRAF/ BENDRAF & CHARMANT & synonyme \\
\hline GRIBI & PARENT & synonyme \\
\hline $\begin{array}{l}\text { MOHAMMED/ } \\
\text { BENMOHAMED/ KARA- } \\
\text { MOHAMED }\end{array}$ & JESUS & noms de prophètes \\
\hline CHEBANI & VIEUX & synonyme \\
\hline CHERCHEM & TABOULÉ & nom d'un plat à base de blé \\
\hline
\end{tabular}

Tableau5

Dans le tableau ci-dessus, nous avons mis en relief les similitudes existantes entre les patronymes mostaganémois et les patronymes français tant sur le plan morphologique, que lexical et sémantique. Jacqueline SUBLET a cerné le patronyme arabe dans son ouvrage Le voile du nom balisant ainsi sa structure intrinsèque. En effet, les spécificités du patronyme arabe ce sont les noms de relation ou « nisba ». Il y a des noms de relation hérités, subdivisés en trois catégories (relatifs à une ethnie, relatifs à un ancêtre et relatifs à un lieu) et des noms de relation acquis. Cette relation crée le surnom ou «laqab ou sobriquet, mais aussi titre de gloire ou titulature) qui tient une si grande place en arabe » (SUBLET, $1990: 155-156)$.

Nous remarquons dans le tableau ci-dessus les caractéristiques communes entre les patronymes mostaganémois et français. Il arrive que nous avons des patronymes synonymes. L'extension des noms relatifs ou nisba à toutes les catégories pour les patronymes mostaganémois est démesurée et s'apparente beaucoup plus à la structure onomastique des patronymes français caractérisés par les surnoms et les sobriquets. 
Onomástica Desde América Latina, n.4, v.2, julho - dezembro, 2021, p.95-109.ISSN 26752719

https://doi.org/10.48075/odal.v0i0.26567

\section{Conclusion}

$\mathrm{Au}$ terme de cette progression, nous sommes arrivés à esquisser les contours d'un symbole identitaire déstructuré de sa forme originelle et greffé sur un autre modèle. Si dans les anciennes colonies françaises d'outre-mer, l'abolition de l'esclavage a entrainé en 1848, l'attribution en masse des noms de famille et a fait naître un ensemble onomastique original fort différent, l'Algérie n'en sera pas épargnée. Cependant, dans l'urgence, les Français ne savaient pas comment nommer cette population indigène, alors ils se sont servis des interprètes juifs et quelques autochtones pour procéder à la nomination des Algériens selon les mêmes principes qui leur ont permis d'acquérir un patronyme en s'adonnant à «des dérives nominatives » (YERMECHE, 2005:20). Comme « le rapport au patronyme reste immuable tout au long de la vie d'un homme »(LEVI-STRAUSS, 2010 : 258), le colonisateur a voulu l'écorcher en tordant cette identité algérienne.

Les spécialistes en anthroponymie sont unanimes quant au calque du patronyme algérien sur un modèle occidental. Le patronyme mostaganémois n'est pas en reste. L'unique caractéristique qui le rattache à la structure onomastique maghrébine, c'est la présence des bases anthroponymiques témoignant autant que possible d'une appartenance jadis bafouée par un occupant assimilateur.

Recebido em 14/12/2020

Aceito em 10/02/2021

Publicado em em 25/08/2021 
Onomástica Desde América Latina, n.4, v.2, julho - dezembro, 2021, p.95-109.ISSN 26752719

https://doi.org/10.48075/odal.v0i0.26567

\section{Bibliographie}

Bengoua Soufiane \& Malek Azzedine.(2001). Les patronymes mostaganémois de 1900 à 1903 Analyse lexico-sémantique, mémoire de licence soutenu, Université de Mostaganem. (Sous la direction de Farid BENRAMDANE)

De Monther Lant, Henry. (1934).Les célibataires. Grasset, France.

Dauzat, Albert.(1994) Dictionnaire étymologique des noms de famille et prénoms de France, Larousse, Paris.

Lacheraf, Mustapha. (1998). Des noms et des lieux, Casbah éditions, Paris.

Le Cour Grandmaison, Olivier (2010), Anatomie d'un monstre juridique : le droit colonial en Algérie et dans l'empire français, La Découverte, Paris, 2010, p. 9.

Sublet, Jacqueline. (1991) Le voile du nom, essai sur le nom propre arabe, PUF écriture, Paris. Levi-Strauss, Claude.(2010). L'identité, PUF, Paris.

Miyamatsu, Hironori.(2002).Surnoms et sobriquets dans l'Ouest de la France médiévale. Une contribution à reconstruire l'image de la bourgeoisie Nouvelle revue d'onomastique, 39-40, p 105-124.

Yermeche, Ouerdia.(2005). L'état civil algérien : genèse d'un processus redénominatif, dans Des noms et des noms Etat civil et anthroponymie en Algérie, (coordonné par Farid Benramdane), CRASC, Oran. 